\title{
Analysis on the Optical Performance and Test Technology of the Multiple Beam of the High-power Laser System
}

\author{
Jianfang $\mathrm{Li}^{1, \mathrm{a}}$, Yanmin Zhou ${ }^{1, \mathrm{~b}}$ \\ ${ }^{1}$ Chongqing College of Electronic Engineering, Chongqing 401331, China \\ alijianfang023023@126.com; 'zhouyanmin023@126.com,
}

Keywords: High power, Laser device, Multiple beams, Optical performance, Test

\begin{abstract}
The laser synchronization to reach targets synchronously is an important index for measuring device integrated performance parameters, also is related to inertial confinement fusion (ICF) physics experiment parameters successful. In the ICF research, the multiple laser pulse need not only waveform and energy, but also need to have a high time consistency. The physics experiment requires multiple laser beams that must arrive at the target, therefore, the precise synchronization of time waveform and synchronous testing and adjusting technology and methods is very important. This paper will discuss the optical performance and testing technology of the high power laser device of multiple beams.
\end{abstract}

\section{Introduction}

Development of high power laser device computer centralized control system, adopts the hierarchical distributed control structure, is domestic first set used in the large-scale scientific computer control system of the device. Currently has carried out a number of key technology research, has completed the simulation control system distributed architecture design, system design, system unit technology research, system design and security interlock system reliability design, etc, these research results for the engineering implementation of the control system provides a reliable technical guarantee. In order to get the maximum output energy as much as possible, in high power laser system is close to optical element damage threshold of flux, which requires the near-field light beam quality has good uniformity. And the light path is very long, optical components of the high power laser device, although the environment requires high clean, high processing requirements are put forward for optical component, but it is still inevitable dirt and element itself defects exist. These scales within a few microns is to several millimeters scattering point, the diffraction effect and nonlinear effect, under the joint action of the greatly reduced after the transfer of near-field beam quality, the normal operation of the threat device. So the scattering point in high power laser system's influence on the beam quality to reduce the operating risk of the device makes sense.

\section{The High Power Laser Device}

The further analysis shows that the high-frequency instability with deviation with running time related systems; the influence is mainly derived from the heating effect of the residual in the sending time of laser depolarization. For III prototype device operating data, established the weighting factor associated with hair time, put forward the related with weighting factor and daily running time and administrative energy output prediction model. Finally through divine III prototype device to establish the model of experimental examination, the results show that compared with the previous single time recursive model, a new model to gain prediction uncertainty has dropped from about $4 \%$ to around $4 \%$, the improvement of accuracy has nearly doubled, it also brings the same degree of optimization for energy balance. The results of the operation have the large solid laser devices. 


\section{The Optical Performance of the High-power Laser System}

The combination of high power drive control requirements, detailed analysis was carried out on the centralized control system structure, the drive control system designed to hierarchical distributed system framework. Computer centralized control system of main implementation unit and the overall control of high-power laser system, complete the data collection, processing, transmission and management. The main features are: the focus of the subsystems of the control and management; Centralized control, testing and environmental monitoring, achieve target control; Physical schemes, experimental parameters definition; Shooting experimental data collection, library; Targeting the analysis of experimental data processing; Target data management, user management system; Power management; Diagnostic test; System safety interlock and alarm, etc.

To meet the demand of high power drive device, to function, cell division, design of control system, can be divided into five major support system (see figure 1), respectively, the computer system, centralized control software system, precise synchronization system, front-end control system and auxiliary system. Each system controlled by the entity unit or functional parts, involves more control equipment types, complicated control parameters and control actions. Equipment and the control object (industrial), and other components, including network equipment, computer, server, data storage, each unit components (such as pulse baseline components, etc.), the power switch machine and all kinds of sensors, etc., they form a sophisticated control system hardware. A large number of applications running on hardware equipment software, tools software and system software, operating system, development platform, database, software design and analysis, software configuration management, network management configuration and application software, etc., these constitute a very large operation control group. Therefore, multi-type is high power drive device of control software and hardware applications, equipment control, system platform, analysis and designs to support the integration of heterogeneous platform framework, such as the computer platform, operating system and programming language support.

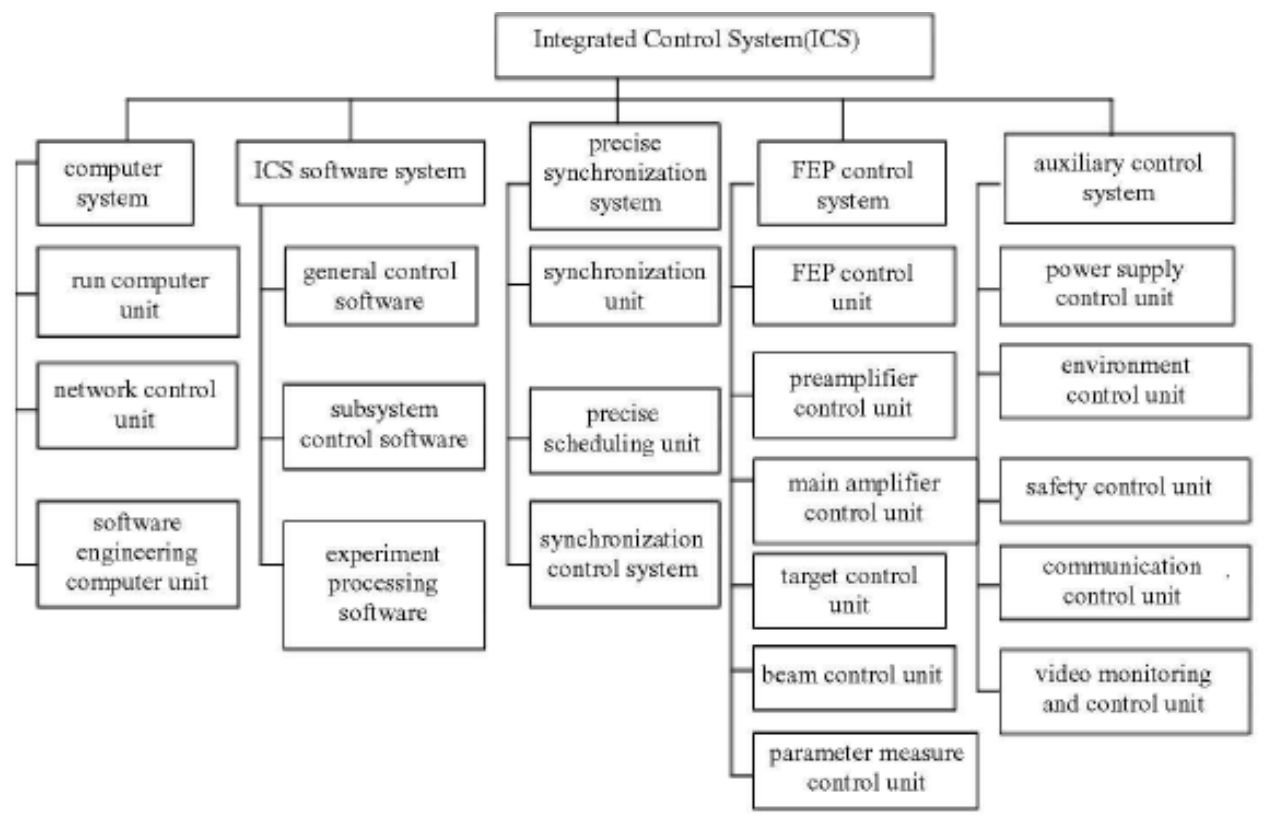

Fig. 1 the Unit Control System

\section{The Testing Techniques and Methods of the High Power Laser Device}

The multi-channel laser output from the oscillator high-power laser system, transmission amplifier, through a series of elements from the final amplifier stage output through two after frequency conversion crystals into three frequency doubling light beam, and then use wedge lens focused beam to the target, each beam of light transmission distance of about $90 \mathrm{~m}$ from the 
oscillator to the target, through detailed regulation of the length of the optical beam multiple beam at the same time to arrive at the target.

To achieve most of the time synchronization laser hair at the same time measurement, at the same time must perform the associated time of multiple beams. A lot of correlation for multiple beam correlation parameters test way, measurement of 16 road laser test points located at different positions of the target chamber $4 \mathrm{~m}$ radius circle, oscilloscope used in the measurement to have as little as possible, and cable length as consistent as possible, considering these factors, using multiple beam series connection layout, associated test diagram as shown in figure 2. Experiments of the communist party of China use five oscilloscope, 2 u $5 \mathrm{~m}$ cable and four power splitters, among them, 1 \# cable for standard time benchmark, each oscilloscope the first channel for four channel oscilloscope measurement signal time benchmark, the fourth channel oscilloscope 1-5 and adjacent another 1 channel of oscilloscope time series association survey, power splitters will signal is divided into two road, all the way, for example, 4 \#, 5 \#, 8 \#, 9 \#, 12 \# and 13 \#, 16 \# and 17 \# two cable is a signals through the two way power splitters, respectively the fourth and the first channel access two oscilloscope and the same signal correlation in two oscilloscope reiteration, benchmark pulse signal will be a number of oscilloscope time base, and by the same time test data can give a 16-way laser time synchronization.

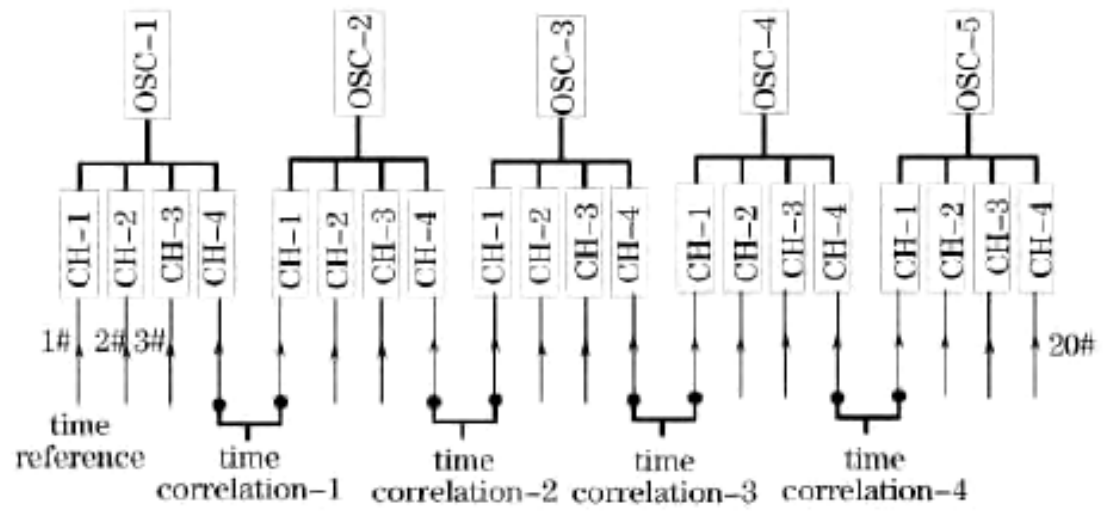

Fig. 2 the Multiple Beam Time Correlation Test Schematic Diagram

Along with the increase of the input energy, the corresponding point location time coordinate move, after the half peak width corresponding to the center of the mobile quantity minimum, half peak width after center position and along the full width half peak position changes are not too much; Given for experimental measurement of flat-roofed pulse power balance is mainly concerned with flat top part of the pulse.

In the experiments, the use of 20 cable material, length and joint should be as consistent as possible, but due to machining error, there is always differences, and, power splitters is only used in correlation channel, therefore, before the trial, the 20 to use $5 \mathrm{~m}$ transmission cables and the inherent time to test the four power splitters, the inherent lag of the cable test diagram as shown in Fig. 3.

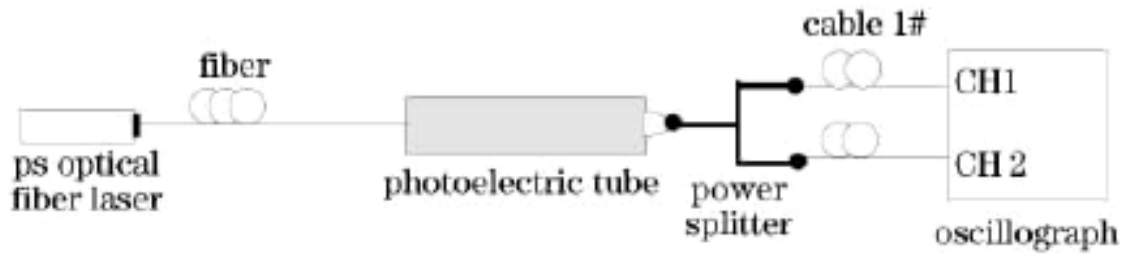

Fig. 3 Relative to the 1 \# Cable Inherent Delay Test Schematic Diagram

Before multiplexing laser power balance test, first using the fast response of double planar diode and wide-band digital oscilloscope test system for eight lasers reach the target chamber window of time synchronization for testing. Test system response time for 110ps; use the light source of repetition frequency output, output about 300ps Gaussian laser pulse width. Place two detectors at the same time in the same way the target chamber window, use the same test two detector response 
and the inherent difference of transmission cables, and then to one of the detector as a benchmark, another probe, respectively, in the rest of the few road test before the target chamber window, multi-channel laser reach the target chamber window of time lag.

For multi-channel high power laser power balance measurement technique, laser power balance measurement of energy test method, time waveform and time synchronization, power balance parameters of the numerical calculation method. In the process of the instantaneous power calculation, the select multiple time of laser pulse waveform half peak width central location as flat-roofed pulse power balance calculation time benchmark, calculate each had three times the frequency of laser output light instantaneous power. Power imbalance between the beams of device, the uncertainty of instantaneous power test result is analyzed. Experiment with photoelectric conversion element, oscilloscope and laser energy calorimeter has realized the time synchronization of multi-channel laser reach the target and power test, synchronous test multi-channel time waveform of pulse peak and the center of the half peak width. Through to the multi-channel laser power test and calculation results, the high power multiple laser power balance are given evaluation method.

\section{Summary}

Through theoretical analysis model of Gaussian random distribution of scattering point, determine after scattering point transmission distance of optical field distribution and transmission distance, the relative position between the scattering area and the scattering points. Numerical simulation results show that in the process of the optical transmission, sub millimeter scale biggest modulation caused the diffraction effect of scattering points can reach 1.4, seriously affected the beam near field uniformity, the surface damage of the optical element after one of the important factors. Long distance transport must also consider nonlinear effect, combination of multiple scattering point diffraction and superposition effect, will seriously affect the beam near field contrast, even cause the beam splitting and small scale self focusing. In order to meet the requirements of components through rate is more than $99.5 \%$, optical element scattering area ratio is less than 0.003 , therefore, to control the optical element surface scattering point number, the number of scattering point especially strict control of large size. Studies have shown that improve the quality of optical element processing and optical path of cleanliness is important to improve the near-field beam quality.

\section{References}

[1] J.F. Seely, C.I. Szabo, P. Audebert, E. Brambrink, E. Tabakhoff, G. E. Holland, L. T. Hudson, A. Henins,P. Indelicato, A. Gumberidze. Hard X-ray spectroscopy of inner-shell K transitions generated by $\mathrm{Me} \mathrm{V}$ electron propagation from intense picoseconds laser focal spots. High Energy Density Physics. 2012(4)

[2] John F. Seely, Glenn E. Holland, Lawrence T. Hudson, Csilla I. Szabo, Albert Henins, Hye-Sook Park,Prav K. Patel, Riccardo Tommasini, J. Martin Laming. K-shell spectra from Ag, Sn, Sm, Ta, and Au generated by intense femtosecond laser pulses. High Energy Density Physics. 2013(1)

[3] L.T. Hudson, R. Atkin, C. A. Back, A. Henins, G.E. Holland, J.F. Seely, C.I. Szabó. X-ray spectroscopy at next-generation inertial confinement fusion sources: Anticipating needs and challenges. Radiation Physics and Chemistry. 2013(11)

[4] T. Ditmire, S. Bless, G. Dyer, A. Edens, W. Grigsby, G. Hays, K. Madison, A. Maltsev, J. Colvin, M.J. Edwards, R.W. Lee, P. Patel, D. Price, B.A. Remington, R. Sheppherd, A. Wootton, J. Zweiback, E. Liang, K.A. Kielty. Overview of future directions in high energy-density and high-field science using ultra-intense lasers[J]. Radiation Physics and Chemistry. 2012(4) 